\title{
Multilayer Transitions in Mixed Spin-3/2 and 1/2 Blume-Capel Model with RKKY Interaction
}

\author{
F. El Hallani, H. Ez-Zahraour*, A. Benyoussef and L. Bahmad

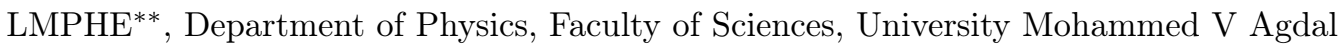 \\ B.P. 1014, Rabat, Morocco
}

(Received January 1, 2011; in final form June 6, 2011)

\begin{abstract}
We studied the effect of the Ruderman-Kittel-Kasuya-Yosida interaction on the multilayer transition and magnetic properties of spins- $3 / 2$ and $1 / 2$ Blume-Capel model in the bilayer separated by a non-magnetic spacer of thickness $(d)$, by using mean-field theory and Monte Carlo simulation. It is found that the multilayer transition temperature depends strongly on the thickness of the non-magnetic layer and the crystal field. Furthermore, the critical thickness of the non-magnetic spacer above which the two magnetic multilayers transit separately depends on the Fermi level and the absolute temperature.
\end{abstract}

PACS: 66.30.Xj, 72.20.Dp, 72.20.My, 72.25.Dc

\section{Introduction}

The discovery of giant magnetoresistance (GMR) [1, 2] has been dramatically improving the technological development, especially increase the capacity of the storage on a disk drive from 1 to 20 gigabit [3]. Either adding the spin degree of freedom to conventional charge-based electronic devices or using the spin alone has the potential advantages of non-volatility, increased data processing speed, decreased electric power consumption, and increased integration densities compared with conventional semiconductor devices [4]. In $\mathrm{Fe} / \mathrm{Si}$ type multilayer, it was observed that the antiferromagnetic coupling strength and the fraction of ferromagnetic coupling due to the Fe percolation mechanism in the non-magnetic inter-layers increase with decrease in temperature [5]. Experimentally, the $\mathrm{Ni}_{80} / \mathrm{Fe}_{20} / \mathrm{Co}_{1} / \mathrm{CuAgAu} / \mathrm{Co}_{2}$ asymmetric sandwiches have been examined for different sublayer thicknesses, it was observed that the ferromagnetic coupling strength oscillates with $\mathrm{CuAgAu}$ thickness with a period of $1 \mathrm{~nm}$ [6]. In multilayer GMR, two ferromagnetic layers are separated by a non-magnetic spacer. It was found that the critical thickness of the non-magnetic layer decreases on increasing the magnetic crystal field and/or the Fermi level $K_{\mathrm{F}}$. Moreover, the multilayer transition temperature undergoes oscillations as a function of the Fermi level and depends strongly on the value of the transverse anisotropy. The multilayer transition temperature decreases when increasing the transverse anisotropy [7].

The spin-1 Blume-Capel film with an alternating crystal field spatially $[8,9]$ has been studied using

\footnotetext{
* corresponding author; e-mail: ezahamid@fsr.ac.ma

** Unité de Recherche Associée au CNRST (URAC)
}

mean-field theory (MFT). However, the effect of the Ruderman-Kittel-Kasuya-Yosida (RKKY) interaction uses the cluster variational method, in pair approximation (CVMPA) [10]. In the case of the antiferromagnetic surface on which a ferromagnetic layer was deposited, it was observed that in the ordered phase depending on the values of the exchange interaction, the antiferromagnetic surface can change to ferromagnetic or ferrimagnetic phase [11]. The layering transitions in the Ising thin films have been studied using many approximate methods, a real space renormalization group [12], MFT [13]. The effect of the non-magnetic thickness on the magnetic properties of an amorphous superlattice has been investigated using effective field theory [14]. However, the effect of the crystal field has been investigated in detail using many approximate methods, namely, mean-field approximation [15], high temperature series expansion [16], constant-coupling approximation [17], Monte Carlo [18] and renormalization-group [19] techniques. Beside, an example of spin-3/2-non-magnetic-spin-3/2 sandwiches has been studied experimentally in the crystalline $\mathrm{Co} / \mathrm{Cu} / \mathrm{Co}$ sandwiches [20] for which the magnetic ordering oscillates from ferromagnetic to the antiferromagnetic by varying the non-magnetic layer $\mathrm{Cu}$ thickness. Furthermore, the magnetoresistance and electrical transport behaviour has been studied experimentally in the case of spin- $3 / 2 /$ spin- $1 / 2$ composite such as $(1-x) \mathrm{La}_{\frac{3}{2}} \mathrm{Ca}_{\frac{1}{2}} \mathrm{MnO}_{3} / x \mathrm{CuO}[21]$. However, the magnetic properties of spin-3/2 Blume-Capel model has been studied numerically using Monte Carlo simulation where the oscillation ferromagnetic antiferromagnetic ordering is observed in Ref. [7].

The purpose of this work is to study, using mean-field theory and Monte Carlo simulation, the effect of RKKY interaction on the multilayer transition and magnetic properties of spin- $3 / 2$ and $1 / 2$ Blume-Capel model in 
the bilayer separated by a non-magnetic spacer of thickness $(d)$.

The paper is organized as follows. In Sect. 2, we present the model and methods. In Sect. 3 we present the results. Finally, Sect. 4 is reserved for conclusion.

\section{Model and methods}

A bilayer Ising system ferromagnetic, consisting of two magnetic blocks $\mathrm{A}$ of spin- $3 / 2$ and $\mathrm{B}$ of spin- $1 / 2$ separated by a non-magnetic layer of thickness $d$. The blocks $\mathrm{A}$ and $\mathrm{B}$ are formed by $N_{1}$ and $N_{2}$ magnetic layers, respectively (Fig. 1). The Hamiltonian of the system is given by

$$
\begin{aligned}
H & =-J \sum_{\langle i, j\rangle} S_{i} S_{j}-\Delta \sum_{i} S_{i}^{2}-\sum_{\left\langle p, p^{\prime}\right\rangle} J_{p p^{\prime}} \sum_{\left\langle i, i^{\prime}\right\rangle} S_{i} \sigma_{i^{\prime}} \\
& -J \sum_{\left\langle i^{\prime}, j^{\prime}\right\rangle} \sigma_{i^{\prime}} \sigma_{j^{\prime}},
\end{aligned}
$$

on the block $\mathrm{A}$ and $\sigma_{i}$ refers to spin- $1 / 2$ with values of $\pm 1 / 2$ on the block $\mathrm{B}$, the $\sum_{\langle i, j\rangle}$ and $\sum_{\left\langle i^{\prime}, j^{\prime}\right\rangle}$ runs over all pairs of nearest-neighbours sites of the blocks A and B, respectively. $\sum_{\left\langle p, p^{\prime}\right\rangle}$ means the summation over all pairs of layers $p(p \in$ block $\mathrm{A})$ and $p^{\prime}\left(p^{\prime} \in\right.$ block $\left.\mathrm{B}\right), \sum_{\left\langle i, i^{\prime}\right\rangle}$ means the summation over axially (with the axis being perpendicular to the non-magnetic layers) connected pairs of sites $i$ and $i^{\prime}$ in layers $p$ and $p^{\prime}$, respectively, and $\sum_{i}$ means the summation over all sites of block A. $J_{p p^{\prime}}$ is the "RKKY-like" coupling, across the non-magnetic layers, between the layers $p$ and $p^{\prime}$ given by

$$
J_{p p^{\prime}}=\frac{J_{0}}{\left(p-p^{\prime}\right)^{2}} \cos \left(k_{\mathrm{F}}\left(p-p^{\prime}\right)\right),
$$

where $k_{\mathrm{F}}$ is the Fermi level, $J_{0}$ is a magnetic coupling constant [22] and $\Delta$ is the crystal field on the block A. The mean-field equations of the magnetization and the quadrupolar moment of each layer $p=1, \ldots, N_{1}$ of spin$3 / 2$ are given respectively by

where $S_{i}$ refers to spin- $3 / 2$ with values of $\pm 3 / 2$ and $\pm 1 / 2$

$$
\begin{gathered}
m_{p}=\frac{3 \exp \left(\frac{9 \Delta \beta}{4}\right) \sinh \left(\frac{3}{2} \beta\left(h_{p}+R_{p}\right)\right)+\exp \left(\frac{\Delta \beta}{4}\right) \sinh \left(\frac{1}{2} \beta\left(h_{p}+R_{p}\right)\right)}{2 \exp \left(\frac{9 \Delta \beta}{4}\right) \cosh \left(\frac{3}{2} \beta\left(h_{p}+R_{p}\right)\right)+2 \exp \left(\frac{\Delta \beta}{4}\right) \cosh \left(\frac{1}{2} \beta\left(h_{p}+R_{p}\right)\right)}, \\
q_{p}=\frac{3 \exp \left(\frac{9 \Delta \beta}{4}\right) \cosh \left(\frac{3}{2} \beta\left(h_{p}+R_{p}\right)\right)+2 \exp \left(\frac{\Delta \beta}{4}\right) \cosh \left(\frac{1}{2} \beta\left(h_{p}+R_{p}\right)\right)}{2 \exp \left(\frac{9 \Delta \beta}{4}\right) \cosh \left(\frac{3}{2} \beta\left(h_{p}+R_{p}\right)\right)+2 \exp \left(\frac{\Delta \beta}{4}\right) \cosh \left(\frac{1}{2} \beta\left(h_{p}+R_{p}\right)\right)}
\end{gathered}
$$

with

$$
\begin{aligned}
& h_{p}=J \sum_{j} S_{j}=J\left(m_{p-1}+4 m_{p}+m_{p+1}\right), \\
& R_{p}=\sum_{p^{\prime}=N_{1}+M+1}^{N_{1}+M+N_{2}} J_{p, p^{\prime}} m_{p^{\prime}} .
\end{aligned}
$$

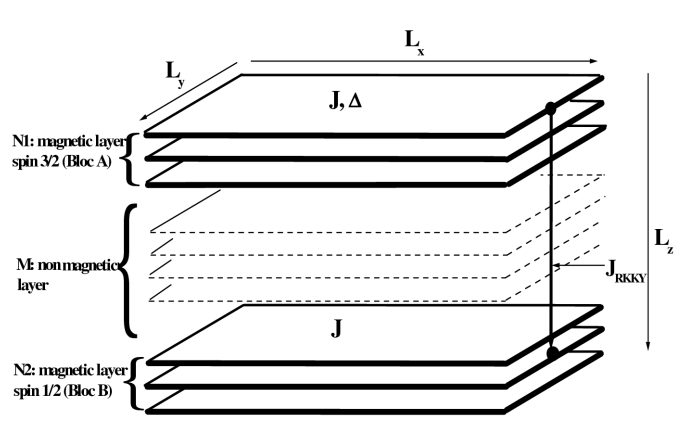

Fig. 1. The illustration of the bilayer separated by non-magnetic layers $M$.

The mean-field equations of the magnetization of each layer $p=N_{1}+M+1, \ldots, N_{1}+M+N_{2}$ for spin $1 / 2$ is given respectively by

$$
\begin{aligned}
& m_{p}=\frac{1}{2} \tanh \left(\frac{\beta}{2}\left(h_{p}^{\prime}+R_{p}\right)\right), \\
& h_{p}^{\prime}=J \sum_{j^{\prime}} \sigma_{j^{\prime}}=J\left(m_{p-1}+4 m_{p}+m_{p+1}\right), \\
& R_{p}=\sum_{p^{\prime}=1}^{N_{1}} J_{p, p^{\prime}} m_{p^{\prime}},
\end{aligned}
$$

$\beta=\frac{1}{k_{\mathrm{B}} T}, k_{\mathrm{B}}$ is the Boltzmann constant, $J$ is the coupling constant, $T$ is the absolute temperature. The reduced magnetizations $m_{\mathrm{A}}$ and $m_{\mathrm{B}}$ of the blocks $\mathrm{A}$ and $\mathrm{B}$, respectively, are given by

$$
\begin{aligned}
& m_{\mathrm{A}}=\frac{1}{N_{1}} \sum_{p=1}^{N_{1}} m_{p}, \\
& m_{\mathrm{B}}=\frac{1}{N_{2}} \sum_{p=N_{1}+M+1}^{N_{1}+M+N_{2}} m_{p},
\end{aligned}
$$

where $m_{p}$ is the reduced magnetization of a layer $p$. Hence, the total magnetization of the system is given by 


$$
m_{\mathrm{T}}=\frac{1}{N_{1}+N_{2}}\left(\sum_{p=1}^{N_{1}} m_{p}+\sum_{p=N_{1}+M+1}^{N_{1}+M+N_{2}} m_{p}\right) .
$$

However, the mean-field reduced free energy of such a system can be written as follows:

$$
\begin{aligned}
F & =-k_{\mathrm{B}} T \sum_{p=1}^{N_{1}+M+N_{2}}\left[\log \left(2 \cosh \left(\frac{\beta}{2} h_{p}^{\prime}\right)\right)\right. \\
& \left.+\log \left(2 \exp \left(\frac{\beta}{2} \Delta\right) \cosh \left(\frac{3 \beta}{2} h_{p}\right)+\cosh \left(\frac{\beta}{2} h_{p}\right)\right)\right] \\
& +\frac{1}{2} \sum_{p=1}^{N_{1}+M+N_{2}}\left[J\left(m_{p} m_{p-1}+4 m_{p}^{2}+m_{p} m_{p+1}\right)\right. \\
& \left.+\sum_{p=N_{1}+M+1}^{N_{1}+M+N_{2}} J_{p p^{\prime}} m_{p} m_{p^{\prime}}+\sum_{p^{\prime}=1}^{N_{1}} J_{p p^{\prime}} m_{p} m_{p^{\prime}}\right] .
\end{aligned}
$$

Using Monte Carlo simulation (Metropolis algorithm) we have computed the total magnetic susceptibility of the system given by

$$
\chi=\frac{1}{k_{\mathrm{B}} T} N_{\mathrm{s}}\left(\left\langle m_{\mathrm{T}}^{2}\right\rangle-\left\langle m_{\mathrm{T}}\right\rangle^{2}\right),
$$

where $N_{\mathrm{s}}$ is the number of spin in each layer.

We performed Metropolis single-spin-flip Monte Carlo simulations $[23,24]$ on our model. Figure 1 has dimensions $L_{x} \times L_{y} \times L_{z}$, with $L_{x}=L_{y}=32$ and $L_{z}$ is the total number of magnetic and non-magnetic layers. Periodic boundary conditions are applied in the lateral ( $x$ and $y$ ) directions and free boundary conditions in the $z$ direction. The flipping attempt was made for every spin on the lattice sites in sequence. At each temperature, $6 \times 10^{3}$ Monte Carlo steps (MCS) have been discarded for equilibration before averaging the physical quantities over the following $3 \times 10^{4} \mathrm{MCS}$.

\section{Results and discussion}

\subsection{Ground state $(T=0 \mathrm{~K})$}

The ground state $(\Delta / J, d)$ phase diagram of the model (Fig. 1) obtained for $N_{1}=N_{2}=3, k_{\mathrm{F}}=0.25, J=1$ and $J_{0}=8$, presented in Fig. 2, shows the existence of two ground state configurations $\left((3 / 2)^{3},(1 / 2)^{3}\right)$ and $\left((3 / 2)^{3},(-1 / 2)^{3}\right)$ alternates between each vertical line for all values of crystal field $(\Delta)$. Moreover, this ground states phase repeats on intervals of length period $(L=$ $\left.2 \pi / k_{\mathrm{F}}\right)$. In the particular case the values of this period is around of 25.142 , where the spins of the sites of all the blocks $\mathrm{A}$ and $\mathrm{B}$ are all equal to $3 / 2,1 / 2$ or $3 / 2,-1 / 2$ respectively.

\subsection{Study at $T>0 \mathrm{~K}$}

First of all, we shall define the phases that we have encountered when investigating this system:

A $(\mathrm{O})$ B $(\mathrm{O})$ : the two magnetic blocks $\mathrm{A}$ and $\mathrm{B}$ are ordered. A $(\mathrm{O}) \mathrm{B}(\mathrm{D})$ : the magnetic block $\mathrm{A}$ is ordered

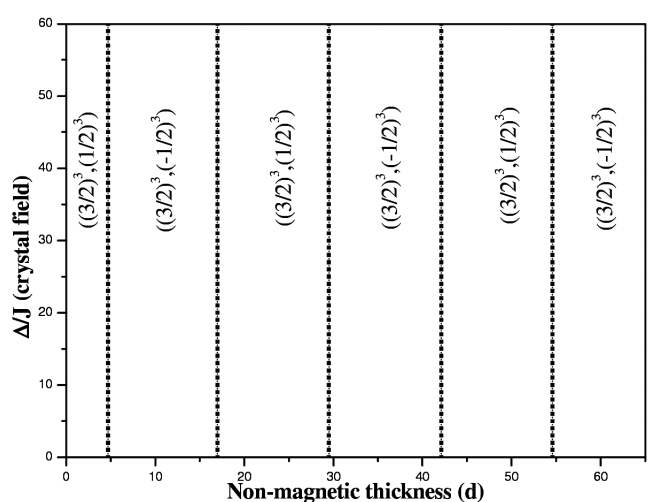

Fig. 2. The ground state phase diagram of the bilayer spin-3/2 and spin- $1 / 2$ Ising model for: $N_{1}=N_{2}=3$, $k_{\mathrm{F}}=0.25, J_{0}=8, J=1$.

and the magnetic block B is disordered. A (D) B (D): the two magnetic blocks $\mathrm{A}$ and $\mathrm{B}$ are disordered.

In this section, we have studied the effect of RKKY interaction (by varying the non-magnetic spacer of thickness $d$ ) and the crystal field on the transition temperature and the magnetic properties of the magnetic layers (blocks A and B). So that seeing the influence of the crystal field on the transition temperature of model (Fig. 1), we have studied, using mean-field theory, the behaviour of the magnetization as a function of the temperature for different values of $\Delta$ (crystal field on the block $\mathrm{A}$ ) for $N_{1}=N_{2}=3, k_{\mathrm{F}}=0.25$ and $d=3$.

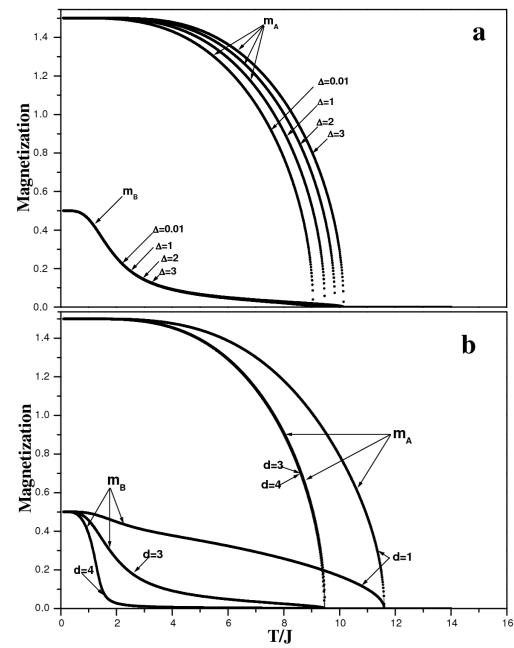

Fig. 3. The dependence of the magnetization as a function of the temperature for $N_{1}=N_{2}=3, k_{\mathrm{F}}=0.25$, $J_{0}=8, J=1$ : (a) several values of $\Delta$ and (b) several values of $d$ (the non-magnetic layer thickness).

It has been observed that the transition temperature of two blocks $T_{\mathrm{c}}^{\mathrm{A}}$ and $T_{\mathrm{c}}^{\mathrm{B}}$ increase on increasing the crystal field $\Delta$ (Fig. 3a). Moreover, Fig. 3b shows that the transition temperature of the multilayer transition decreases on increasing the thickness of non-magnetic layers $d$, for sufficiently large values of $d(d=3)$. 


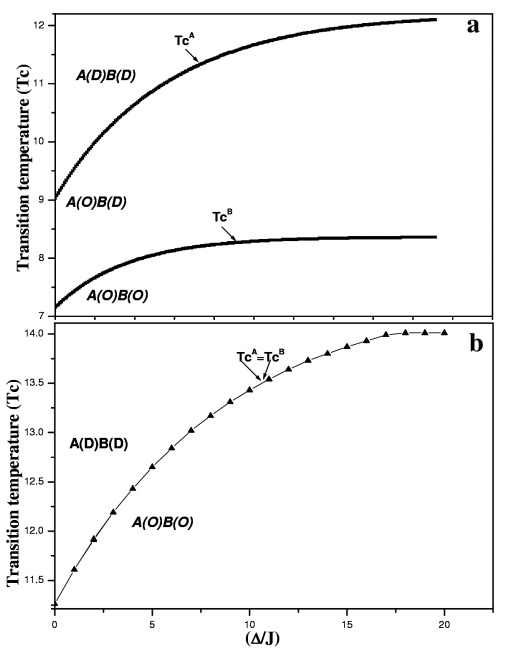

Fig. 4. The behavior of the critical temperature according to the crystal field for several values of $d$ (the non-magnetic layer thickness) for $N_{1}=N_{2}=3, k_{\mathrm{F}}=$ $0.25, J_{0}=8, J=1$ : (a) $d=3$, (b) $d=1$. D and $\mathrm{O}$ indicate disordered and ordered ferroagnetic phases, respectively.

The multilayers block A and block B exhibit an order-disorder transition at different critical temperatures (i.e. the two magnetic blocks A and B transit separately) (Fig. 4a). For small thickness of the non-magnetic spacer $d(d=1)$, the multilayers block A and block B exhibit an order-disorder transition at the same temperature (i.e. $T_{\mathrm{c}}^{\mathrm{A}}=T_{\mathrm{c}}^{\mathrm{B}}$, the two magnetic blocks $\mathrm{A}$ and $\mathrm{B}$ transit simultaneously) (Fig. 4b). Indeed, due to small thickness of the non-magnetic spacer $d$ the RKKY interaction becomes strongly dominant in comparison with the thermal fluctuations and then the magnetism in block B is reinforced by the magnetism of block A via RKKY interaction. Moreover, by using Monte Carlo simulation (the Metropolis algorithm), we have studied the total magnetic susceptibility and the magnetization of the blocks A and B. It is found that the susceptibility exhibits only one peak at the transition temperature for small thickness of the non-magnetic spacer $(d=1.5)$, where the RKKY interaction is more important than the thermal fluctuations.

Then the magnetic blocks A and B exhibit an order-disorder transition at the same temperature (Fig. 5a). In the case of a sufficiently large non-magnetic spacer, the thermal fluctuations become more important than the RKKY exchange interaction, and the magnetism of the system is governed by the competition between crystal field and thermal fluctuations. However, the multilayers block A and block B exhibit an order-disorder transition at different critical temperatures. Indeed, for $d=4$, the susceptibility presents two peaks at two different critical temperatures. The first peak $\left(T_{\mathrm{c}}^{\mathrm{B}}=1.02\right)$ corresponds to the transition of the magnetic block $\mathrm{B}$, while the second peak $\left(T_{\mathrm{c}}^{\mathrm{A}}=5.67\right)$ corresponds to the transition of

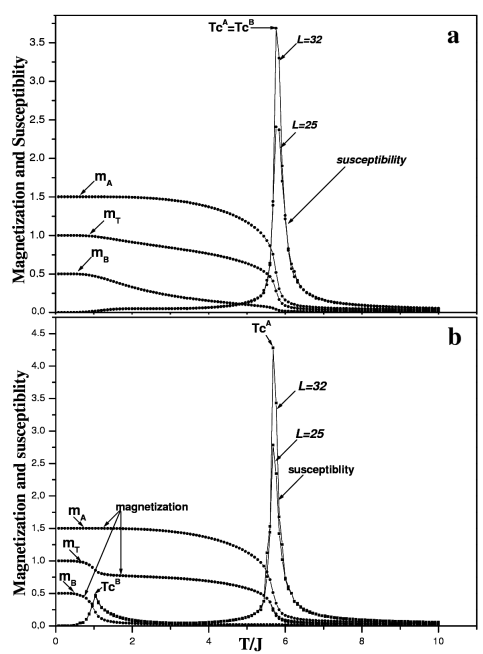

Fig. 5. The dependence of the susceptibility and magnetization as a function of the temperature obtained within Monte Carlo simulation, for $N_{1}=N_{2}=3$, $k_{\mathrm{F}}=0.25, J_{0}=8, J=1, \Delta=1:$ (a) $d=1.5, L=25$ and $L=32$; (b) $d=4, L=25$ and $L=32$.

the magnetic block A (Fig. 5b). These results are qualitatively in good agreement with those obtained within MFT.

However, in Fig. 6a and Fig. 6b, we have plotted the variation of the transition temperature of the multilayer transition as a function of non-magnetic layer thickness within mean-field theory, and in Fig. 6c within Monte Carlo simulation. It is clear that we have three phases of the system for different values of $d$ :

- First phase $\mathrm{A}(\mathrm{O}) \mathrm{B}(\mathrm{O})$ : the two magnetic blocks $\mathrm{A}$ and $\mathrm{B}$ are ordered ferromagnetically or antiferromagnetically depending on the thickness of the non-magnetic spacer accordingly with the RKKY coupling oscillations. This is qualitatively in good agreement with experiment results obtained in Ref. [20];

- Second phase $\mathrm{A}(\mathrm{O}) \mathrm{B}(\mathrm{D})$ : the magnetic block $\mathrm{A}$ is ordered and the magnetic block $\mathrm{B}$ is disordered;

— Third phase A(D) B(D): the two magnetic blocks A and $\mathrm{B}$ are disordered.

Moreover, the multilayer transition, where A and B transit separately from the ordered phase to the disordered one, occurs above a certain non-magnetic layer thickness $\left(d_{\mathrm{c}}\right)$. This is qualitatively in good agreement with results obtained in Ref. [7], but quantitatively we have found that the critical thickness $d_{\mathrm{c}}=1.85$ (Fig. 6a) is lower than the one obtained for spins- $3 / 2$ Blume-Capel model $\left(d_{\mathrm{c}}=5\right)$ in Ref. [7]. Moreover, the critical thickness of non-magnetic spacer above which the two magnetic multilayers transit separately is $d_{\mathrm{c}}=1.44$ within mean-field theory (Fig. 6b) and $d_{\mathrm{c}}=2.99$ within Monte Carlo simulation (Fig. 6c). However, it is clear that the critical thickness from Monte Carlo is twice large as the one determined by MFT. On the other hand, for mean field (Fig. 6b), the curves $T_{\mathrm{c}}^{\mathrm{A}}(d)$ and $T_{\mathrm{c}}^{\mathrm{B}}(d)$ separate at 


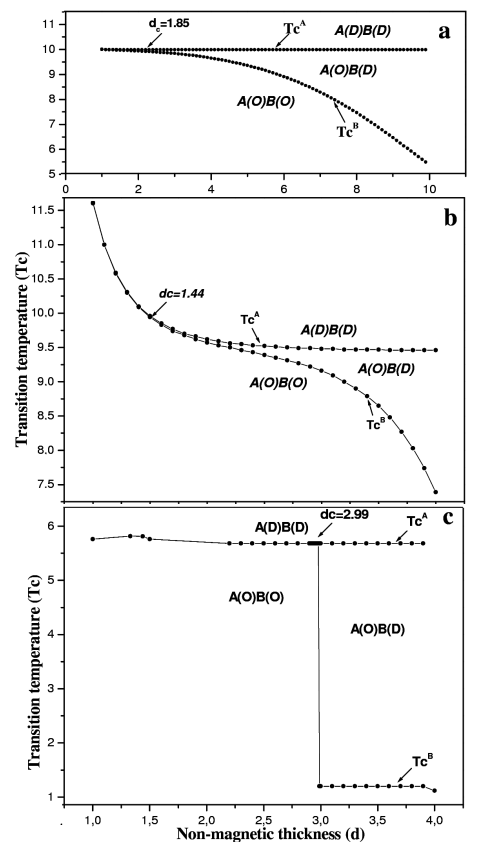

Fig. 6. The behavior of the transition temperature according to the non-magnetic layer of thickness $d$ for $J=1, \Delta=1$ : (a) and (b) correspond to mean field theory with $N_{1}=N_{2}=5, k_{\mathrm{F}}=0.085, J_{0}=1$ and $N_{1}=N_{2}=3, k_{\mathrm{F}}=0.25, J_{0}=8$ respectively, while (c) correspond to Monte Carlo simulation with $N_{1}=N_{2}=3, k_{\mathrm{F}}=0.25, J_{0}=8$.

the critical $d_{\mathrm{c}}$ and change continuously. On the contrary, the phase diagram presented in Fig. 6c shows that the critical temperature of the block $\mathrm{B} T_{\mathrm{c}}^{\mathrm{B}}$ changes discontinuously at the critical thickness $d_{\mathrm{c}}$. This is due to the fact that the Monte Carlo simulation takes into account the correlation between the spins of the blocks A and B. However for sufficiently large thickness $\left(d>d_{\mathrm{c}}\right)$ the correlation between blocks $\mathrm{A}$ and $\mathrm{B}$ becomes negligible and then the two blocks transit separately, while the mean field approximation neglects all correlations between spins. Thus by using Monte Carlo, we need very large thickness of non-magnetic spacer to decouple magnetically the two magnetic blocks.

To illustrate the jump of the critical temperature $T_{\mathrm{c}}^{\mathrm{B}}$ of the block $\mathrm{B}$ as a function of the thickness $d$ of the non-magnetic spacer, we have plotted, in Fig. 7, the magnetizations and magnetic susceptibility of the system in the case of a thickness $(d=2.95)$ slightly smaller than $d_{\mathrm{c}}$ (Fig. 7a) and the case of a thickness $(d=3)$ slightly greater than $d_{\mathrm{c}}$ (Fig. 7b). It is clear that the two blocks transit at the same temperature for $d<d_{\mathrm{c}}$, while for $d>d_{\mathrm{c}}$ the two blocks transit separately. This is in good agreement with the phase diagram established in Fig. 6c.

\section{Conclusions}

In this work, we have applied MFT and Monte Carlo simulation to the study of the magnetic properties of

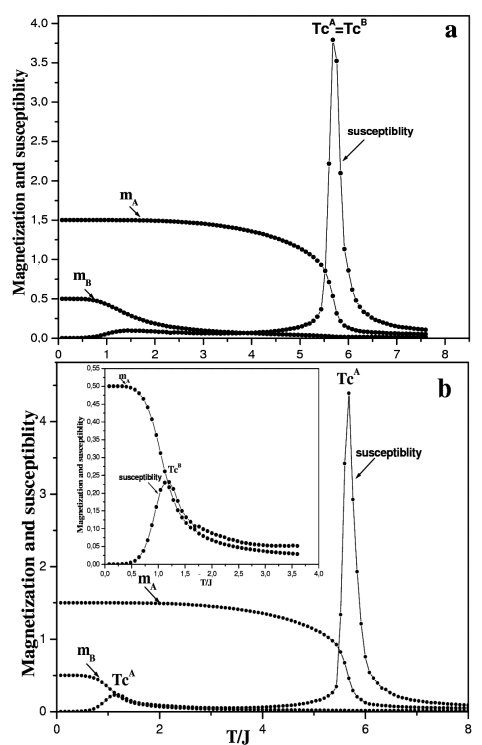

Fig. 7. The dependence of the susceptibility and magnetization as a function of the temperature obtained within Monte Carlo simulation, for $N_{1}=N_{2}=3$, $k_{\mathrm{F}}=0.25, J_{0}=8, J=1, \Delta=1:$ (a) $d=2.95, L=32$, (b) $d=3, L=32$. The inset in (b) is given in large scale to show better the transition associated to the block B.

spin-3/2 and $1 / 2$ Blume-Capel model in the bilayer separated by a non-magnetic spacer of finite thickness. We have shown that the transition temperature of multilayer transition depends strongly of the thickness of the non-magnetic spacer and/or the crystal field. We have shown the existence of a critical thickness of non-magnetic spacer above which the magnetic multilayer transit separately. Below this critical thickness the system exhibits a ferromagnetic antiferromagnetic ordering oscillation depending on the value of the thickness of the non-magnetic spacer. The critical thickness computed by Monte Carlo simulation is lower than the one obtained within mean-field approximation.

\section{References}

[1] M.N. Baibich, J.M. Broto, A. Fert, F. Nguyen Van Dau, F. Petroff, P. Etienne, G. Creuzet, A. Friederich, J. Chazelas, Phys. Rev. Lett. 61, 2472 (1988).

[2] G. Binasch, P. Grunberg, F. Saurenbach, W. Zinn, Phys. Rev. B 39, 4828 (1989).

[3] G.A. Prinz, Science 282, 1660 (1998).

[4] S.A. Wolf, D.D. Awschalom, R.A. Buhrman, J.M. Daughton, S. von Molnár, M.L. Roukes, A.Y. Chtchelkanova, D.M. Treger, Science 294, 1488 (2001).

[5] J. Kohlhepp, F.J.A. den Broeder, J. Magn. Magn. Mater. 156, 261 (1996).

[6] T. Lucinski, A. Hutten, H. Bruckl, S. Heitmann, T. Hempel, G. Reiss, J. Magn. Magn. Mater. 269, 78 (2004). 
[7] N. Tahiri, H. Ez-Zahraouy, A. Benyoussef, Physica A 388, 3426 (2009); N. Tahiri, H. Ez-Zahraouy, A. Benyoussef, Chin. Phys. B 20, 017501 (2011).

[8] H. Ez-Zahraouy, A. Kassou-Ou-Ali, Phys. Rev. B 69, 064415 (2004).

[9] S.M. Pittman, G.G. Batrouni, R.T. Scalettar, Phys. Rev. B 78, 214208 (2008).

[10] T. Balcerzak, J.W. Tucker, J. Magn. Magn. Mater. 278, 8795 (2004)

[11] Y. Belmamoun, H. Ez-Zahraouy, M. Kerouad, J. Magn. Magn. Mater. 321, 635639 (2009).

[12] A. Benyoussef, H. Ez-Zahraouy, Physica A 206, 196 (1994).

[13] T. Kaneyoshi, J.C. Chen, J. Magn. Magn. Mater. 98 201 (1991)

[14] A. Benyoussef, H. Ez-Zahraouy, Phys. Rev. B 52, 6 (1995).

[15] M. Blume, Phys. Rev. 141, 517 (1966); H.W. Capel, Physica 32, 966 (1966).

[16] P.F. Fox, D.S. Gaunt, J. Phys. C 5, 3085 (1972).
[17] M. Tanaka, K. Takahashi, Phys. Status Solidi B 93, 85 (1979).

[18] W. Selke, J. Yeomans, J. Phys. A 16, 2789 (1983); M. Deserno, Phys. Rev. E 56, 5204 (1997).

[19] A.N. Berker, M. Wortis, Phys. Rev. B 14, 4946 (1976); H. Dickinson, J. Yeomans, J. Phys. C 16 345 (1983).

[20] A. Cebollada, R. Miranda, C.M. Schneider, P. Schuster, J. Kirschner, J. Magn. Magn. Mater. 102, 25 (1991).

[21] J.H. Miao, L. Yuan, Y.Q. Wang, J.L. Shang, G.Q. Yu, G.M. Ren, X. Xiao, S.L. Yuan, Mater. Lett. 60, 2214 (2006).

[22] M.A. Ruderman, C. Kittel, Phys. Rev. 96, 99 (1954); T. Kasuya, Prog. Theor. Phys. 16, 45 (1956); K. Yosida, Phys. Rev. 106, 893 (1957).

[23] K. Binder, in: Phase Transitions and Critical Phenomena, Vol. 5b, Eds. C. Domb, M.S. Green, Academic, New York 1976.

[24] D.P. Landau, Phys. Rev. B 13, 2997 (1976). 